F.M. Zhou*, Q.Y. Zhang, M.X. Shi, H. Li and J.W. Guo

\title{
Structural design and wear properties of TIG arc brazing tin-based babbit to mild steel
}

https://doi.org/10.1515/htmp-2016-0240

Received November 27, 2016; accepted August 15, 2017

\begin{abstract}
The tin-based babbit with different layer thickness was bonded to mild steel via TIG arc brazing. The microstructure, microhardness, wear properties and phase formation mechanism near the interface of the arc brazed layer were investigated by the optical microscope (OM), X-ray diffraction (XRD), high temperature friction and wear testing machine (HTFWT), laser scanning confocal microscope (LSCM), electron microscope (SEM) and energy dispersion spectrum (EDS). It can be found that in the arc brazing seam region, the thinner layer made it possible to form larger size SbSn monotectic phase that connected together and some Fe-Sn IMCs were formed near the interface. So that when the layer thickness was $0.5 \mathrm{~mm}$, the microhardness of arc brazing tinbased babbit layer was the largest and the antifriction property was the best. Moreover, on the brazing tin-based babbit interface, Fe elements were transited and diffused from mild steel into the brazing seam region. Element concentration of Fe was high near the steel interface and formed concentration gradient in the liquid metal layer, which was helpful to promote the metallurgical reaction. Phase constituents of the brazed joint interface were Fe, $\mathrm{Fe}_{3} \mathrm{Sn}, \mathrm{FeSn}$ and $\mathrm{FeSn}_{2}$, formed in the metallurgy of $\mathrm{Fe}$ from mild steel and the Sn from tin-based babbit.
\end{abstract}

Keywords: tin-based babbit, TIG arc brazing, antifriction property, IMCs

\section{Introduction}

The tin-based babbit is widely used as antifriction coatings for sliding bearings, since they are superior to other antifriction alloys in antifriction properties, what's more, the tin-based babbit is usually bonded to bush substrate in order to obtain the better comprehensive performance.

*Corresponding author: F.M. Zhou, Jiangsu Province Key Laboratory of Advanced Welding Technology, Jiangsu university of Science and Technology, Zhenjiang, 212003, China,

E-mail: fangmingzhou@just.edu.cn

Q.Y. Zhang, M.X. Shi, H. Li, J.W. Guo, Jiangsu Province Key Laboratory of Advanced Welding Technology, Jiangsu university of Science and Technology, Zhenjiang, 212003, China
Many achievements [1-4] have shown that there are some disadvantages in the tin-based babbit layer which is formed by pouring, such as volatile chemical composition, phase separation of the process of crystallization and formation of inclusions, leading to "stretching" of the alloy on the shaft. To exclude these disadvantages, gas surfacing and spraying have been successfully applied, in which metal atomization is done by propane-air combustion product or arc [2]. However, these methods as the same as casting always need to hang tin before process, which reduce productivity apparently. Therefore, it is receiving a remarkable attention to prepare tin-based babbit antifriction layer by new technology process.

TIG arc brazing technology has been successfully used to join dissimilar metal because of its lower welding heating input, better protection effect, smaller heat affected zone and welding deformation [5-7]. In this technique, filler wire metals would be melted by arc and spread to the infusible iron substrate surface, metallurgical bonding between filler metal and iron substrate can be obtained. However, there are some hard intermetallic compounds (IMCs) which are formed mainly between $\mathrm{Fe}$ and $\mathrm{Sn}$ atoms $[8,14]$. At present, there are few reports about wear property of these IMCs [9]. Besides, the layer thickness of tin-based babbit by the method of casting, gas surfacing and spraying ranges from 1 to $12 \mathrm{~mm}$ or more. With the rapid development of industry, ships, vehicles, aerospace and other heavy machinery industry have witnessed higher requirements of green manufacturing [10] for the antifriction layers thickness of the sliding bearings.

In this article, the layer thickness of tin-based babbit will be designed and prepared through TIG arc brazing, and the wear properties of which will be studied especially the wear property of IMCs. And it will have certain theoretical and practical significance.

\section{Experimental}

The tin-based babbit wire with diameter of $4 \mathrm{~mm}$ and Q235B steel with thickness of $8 \mathrm{~mm}$ were used for the study and their chemical composition were shown in 
Tables 1 and 2. And the Argon with the concentration of $99 \%$ was used as the shielding gas. Besides, the steel substrate was cut into the size of $50 \times 50 \mathrm{~mm}$ and the surface of which was cleaned before the TIG arc brazing. The substrate was fixed and welding schematic diagram was shown in Figure 1. The welding parameters were shown in Table 3.

Table 1: Chemical constitution of the ZChSnSb11-6 (wt\%).

\begin{tabular}{lrr}
\hline Sb & Cu & Sn \\
\hline $10-12$ & $5.5-6.5$ & bal \\
\hline
\end{tabular}

Table 2: Chemical constitution of the Q235B(wt\%).

\begin{tabular}{lrrrrr}
\hline C & Mn & Si & S & P & Fe \\
\hline $0.14-0.22$ & $0.30-0.65$ & $\leq 0.30$ & $\leq 0.05$ & $\leq 0.045$ & bal \\
\hline
\end{tabular}

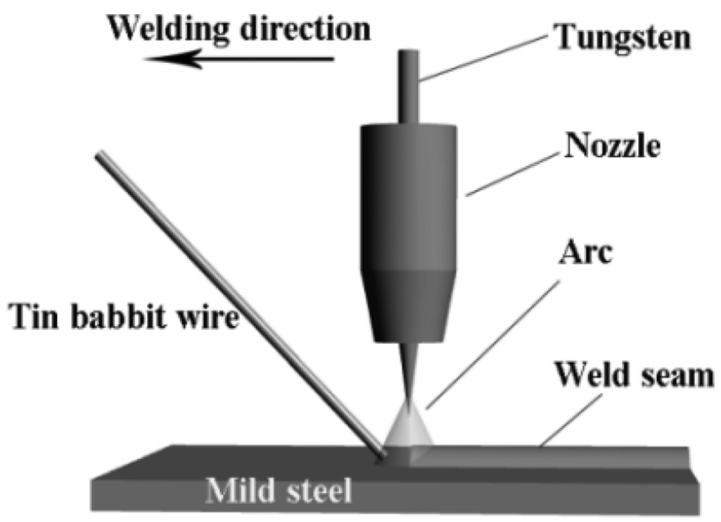

Figure 1: Schematic of Babbitt metal arc surfacing.

Table 3: Welding parameters of TIG arc brazing.

\begin{tabular}{lcrr}
\hline Current $(A)$ & Voltage $(V)$ & $\begin{array}{r}\text { Welding speed } \\
(\mathrm{mm} / \mathrm{min})\end{array}$ & $\begin{array}{r}\text { Gas flow } \\
(\mathrm{L} / \mathrm{min})\end{array}$ \\
\hline $70-90$ & $10-15$ & $80-100$ & 15 \\
\hline
\end{tabular}

The weld seams were cut into the size of $15 \times 15 \times 10 \mathrm{~mm}$ as samples. The layer thicknesses of samples are respectively about $2 \mathrm{~mm}, 1.5 \mathrm{~mm}, 1 \mathrm{~mm}$ and $0.5 \mathrm{~mm}$. The cross-section of samples was pre-grinded, polished, and then corroded by 3-5\% nitric acid alcohol corrosion. The microhardness was carried out by MH-5 microhardness tester with the applied load of $25 \mathrm{~g}$ and dwelling time of $10 \mathrm{~s}$.
The wear properties were carried out under the dry friction. Wear rate is based on the formula: $\mathrm{I}=\Delta \mathrm{V} / \Delta \mathrm{t} \cdot \mathrm{F}$, where $\mathrm{I}$ is wear rate, $\Delta \mathrm{V}$ is volume wear loss, $\Delta \mathrm{t}$ is time and $\mathrm{F}$ is load. The specimens with a friction radius of $4 \mathrm{~mm}$ run in HTFWT. The friction ball was made from $\mathrm{GCr} 15$ with the diameter of $3 \mathrm{~mm}$ and its chemical composition is shown in Table 4. Applied load was $200 \mathrm{~g}$ and speed was $300 \mathrm{rpm}$. The experimental time was 30 minutes and experimental temperature was $25^{\circ} \mathrm{C}$. The friction and wear diagram was shown in Figure 2.

Table 4: Chemical composition of the GCr15 (wt\%).

\begin{tabular}{lr}
\hline $\mathrm{C}$ & $\mathbf{0 . 9 5 - 1 . 0 5}$ \\
\hline $\mathrm{Mn}$ & $0.25-0.45$ \\
$\mathrm{Si}$ & $0.15-0.35$ \\
$\mathrm{~S}$ & $\leq 0.025$ \\
$\mathrm{P}$ & $\leq 0.025$ \\
$\mathrm{Cr}$ & $1.40-1.65$ \\
$\mathrm{Mo}$ & $\leq 0.10$ \\
$\mathrm{Ni}$ & $\leq 0.30$ \\
$\mathrm{Cu}$ & $\leq 0.25$ \\
$\mathrm{Ni}+\mathrm{Cu}$ & $\leq 0.50$ \\
$\mathrm{Hv}$ & 270 \\
\hline
\end{tabular}

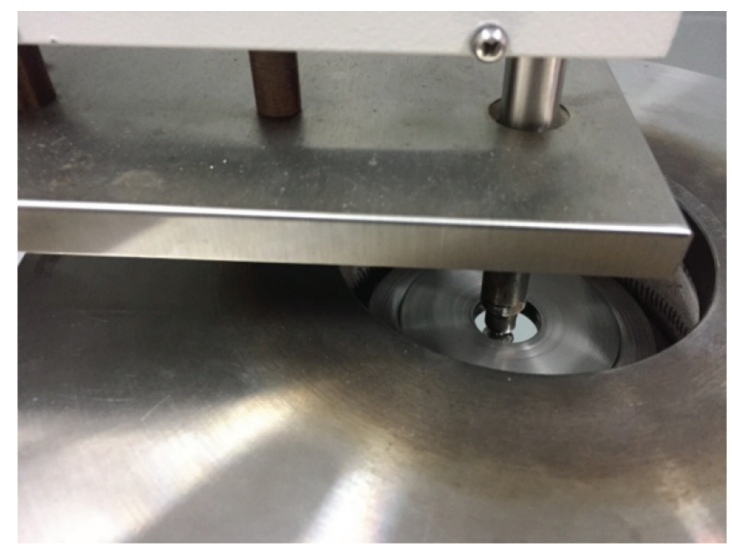

Figure 2: Friction and wear schematic diagram.

Microstructure of the tin-based babbit was studied by OM, XRD, SEM and EDS. The wear mechanism was analyzed through LSCM.

\section{Results and discussion}

\section{Structure morphology and hardness}

It is necessary for welding to study its weld formation firstly due to it plays an important role in material's microstructure 
and property. Figure 3 showed the weld appearance of TIG arc brazing tin-based babbit, it can be seen that the weld formation was better and smooth. Figure 4 showed the different layer thickness of TIG arc brazing tin-based babbit. As we can see from the figure, the straight interface can be obtained and local welding-brazing occurred. As we all know, the hardness of metal materials indirectly reflected their wear properties. Therefore, the microhardness of different layer thickness was tested and the results were shown in Figure 5. The microhardness was approximately constant for the thickness over 0.5 $\mathrm{mm}$, while the layer thickness was $0.5 \mathrm{~mm}$, microhardness was the largest.

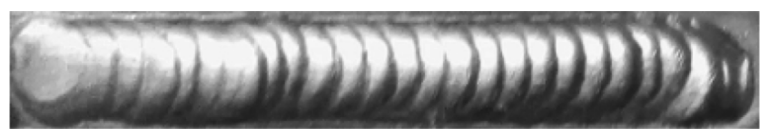

Figure 3: Weld appearance of TIG arc brazing tin-based babbit.

\section{Wear property of different layer thickness}

Figure 6 showed the wear behavior of TIG arc brazing tinbased babbit at different layer thickness. It can be seen from Figure 6(a) that the wear depth was approximately constant for layer thickness over $1 \mathrm{~mm}$, while the wear depth reduced apparently for layer thickness below $1 \mathrm{~mm}$. It is analyzed that when the layer thickness was below $1 \mathrm{~mm}$, the surface was harder so that it can resist the

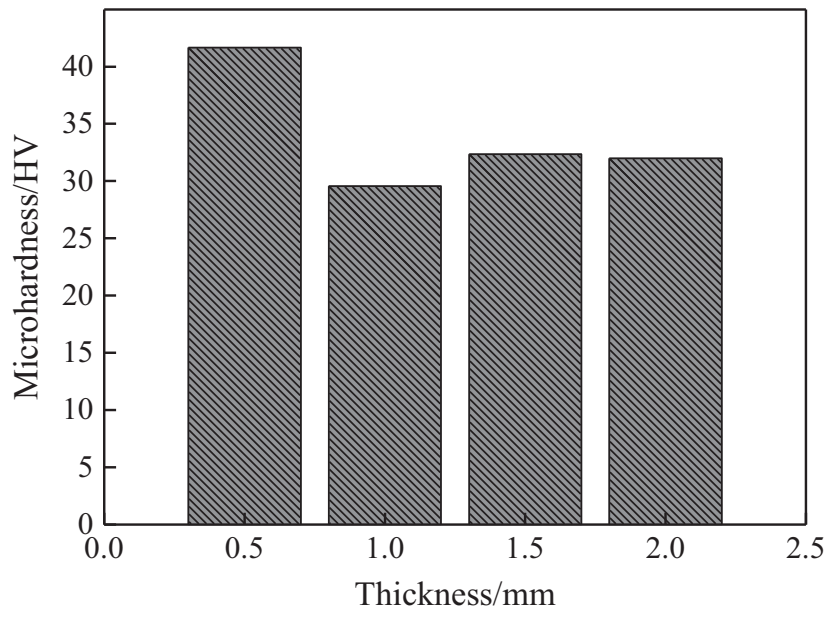

Figure 5: Microhardness of different layer thickness.

deformation due to the dwelling load better than that over $1 \mathrm{~mm}$. Therefore, when the layer thickness was $0.5 \mathrm{~mm}$, the wear depth was the minimum. It can be seen from Figure 6 (b), with the layer thickness of TIG arc brazing tin-based babbit decreasing, the wear rate was obviously decreased. When the layer thickness was $0.5 \mathrm{~mm}$, the wear rate is the minimum value, which is about $1 / 6$ of $2 \mathrm{~mm}$. Therefore, the wear property of tin-based babbit obtained by means of TIG arc brazing especially service life can be improved by decreasing the layer thickness.

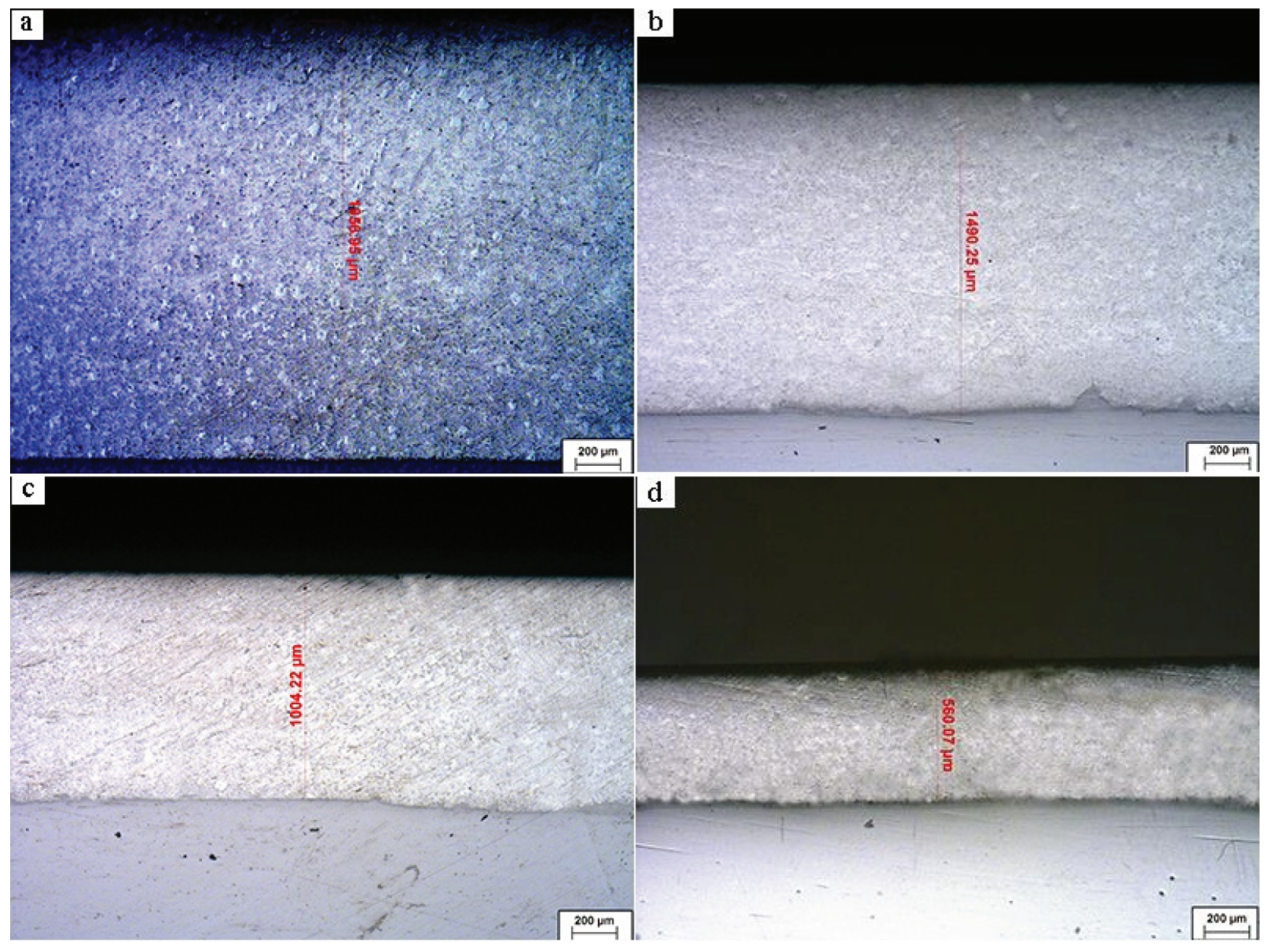

Figure 4: Structure morphology of different layer thickness: a) $2 \mathrm{~mm}$, b) $1.5 \mathrm{~mm}$, c) $1 \mathrm{~mm}$, d) $0.5 \mathrm{~mm}$. 

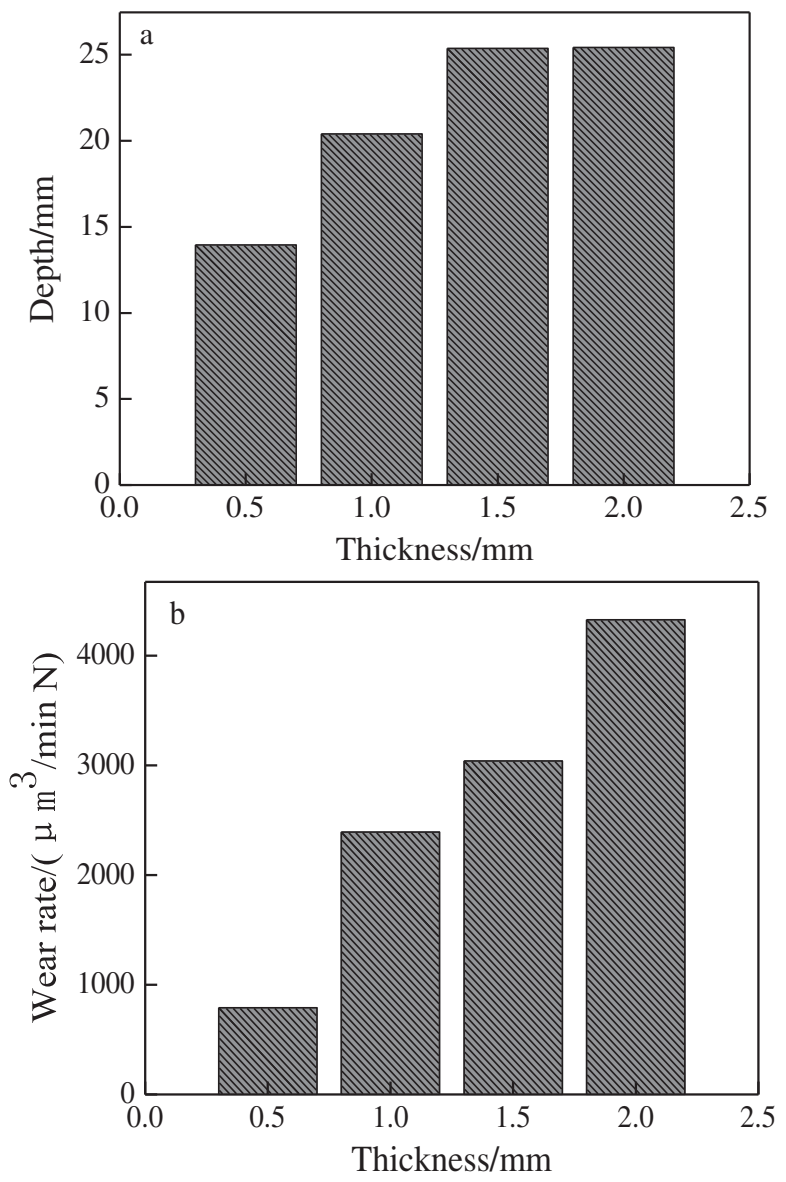

Figure 6: Wear behavior of different layer thickness.
To study the wear mechanism of tin-based babbit at different layer thickness, the wear morphology was observed by Laser scanning confocal microscope and the results were shown in Figure 7. The microstructure of tin-based babbit consisted of a soft matrix with inclusions of the reinforcing phases. The matrix was the phase $\alpha$ (copper and antimony solution in Sn matrix), ensuring efficient running-in and also the special surface microrelief, improving the supply of oil to the friction areas and heat removal from them. The hard inclusions of the phase $\beta$ (SnSb compound) and phase $\varepsilon\left(\mathrm{Cu}_{6} \mathrm{Sn}_{5}\right.$ compound) resulted in high wear resistance $[2,3]$. At real stable manner, the Sn-based solid solution was soft and even melted because of the large amount of friction heat and the furrow would be formed under the action of abrasive wear [11-13]. Also, the soft Sn-based solid solution was easily to take place plastic deformation so that the fluent tin-based babbit was extruded out of the surface, as shown in Figure $7(\mathrm{a}-\mathrm{c})$. While the hard phase $\beta$ still can maintain the shape of solid particles because of their high melting point but these hard $\mathrm{SbSn}$ and $\mathrm{Cu}_{6} \mathrm{Sn}_{5}$ compound particles would shattered and left a hole under the action of friction. And the wear width decreased with the layer thickness decreasing.

In order to further analyze the wear mechanism of TIG arc brazing tin-based babbit at different layer thickness, the wear morphology of grinding ball was tested through LSCM and the results were shown in Figure 8. It can be seen from
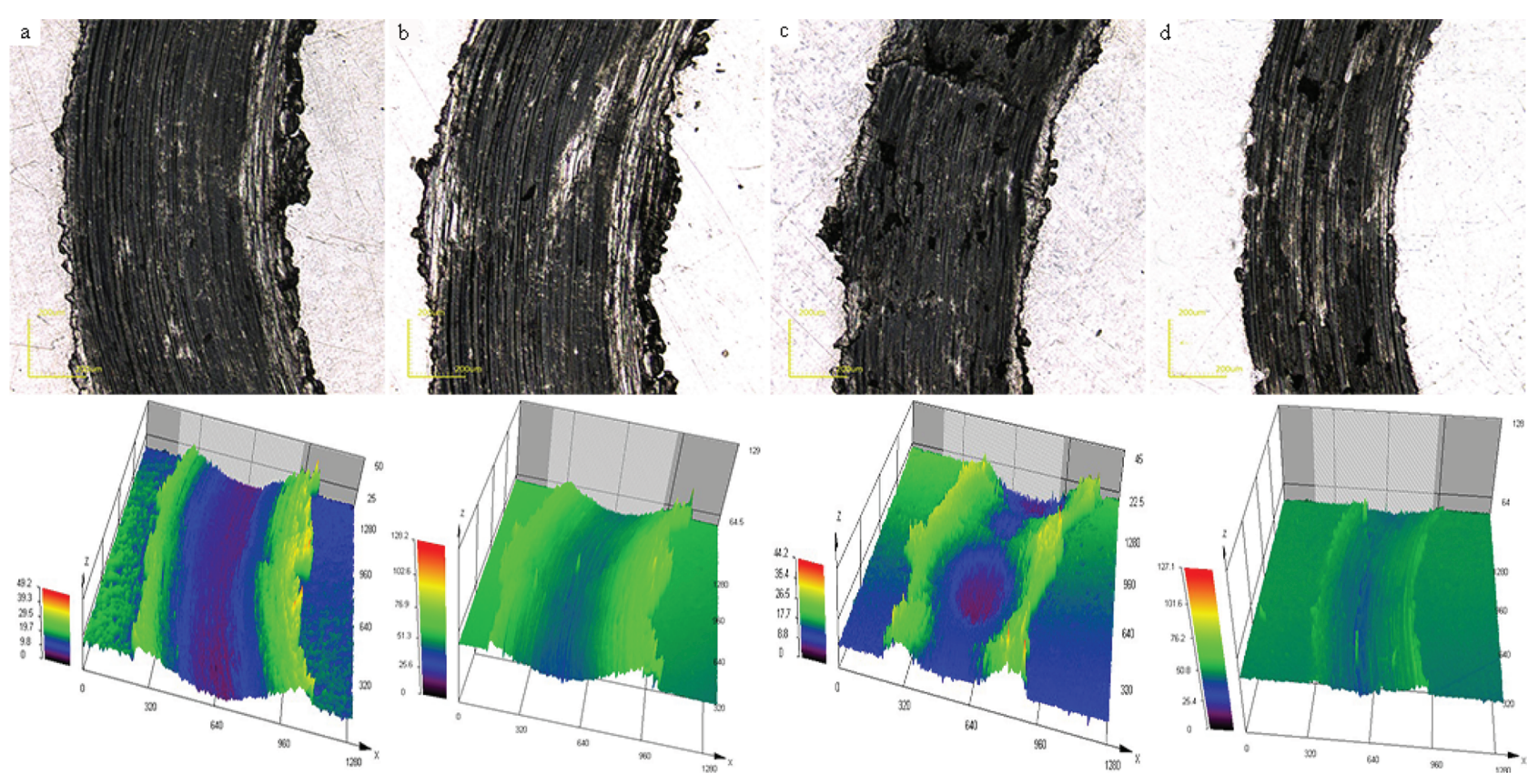

Figure 7: Wear morphology of different layer thickness: a) $2 \mathrm{~mm}$, b) $1.5 \mathrm{~mm}$, c) $1 \mathrm{~mm}$, d) $0.5 \mathrm{~mm}$. 


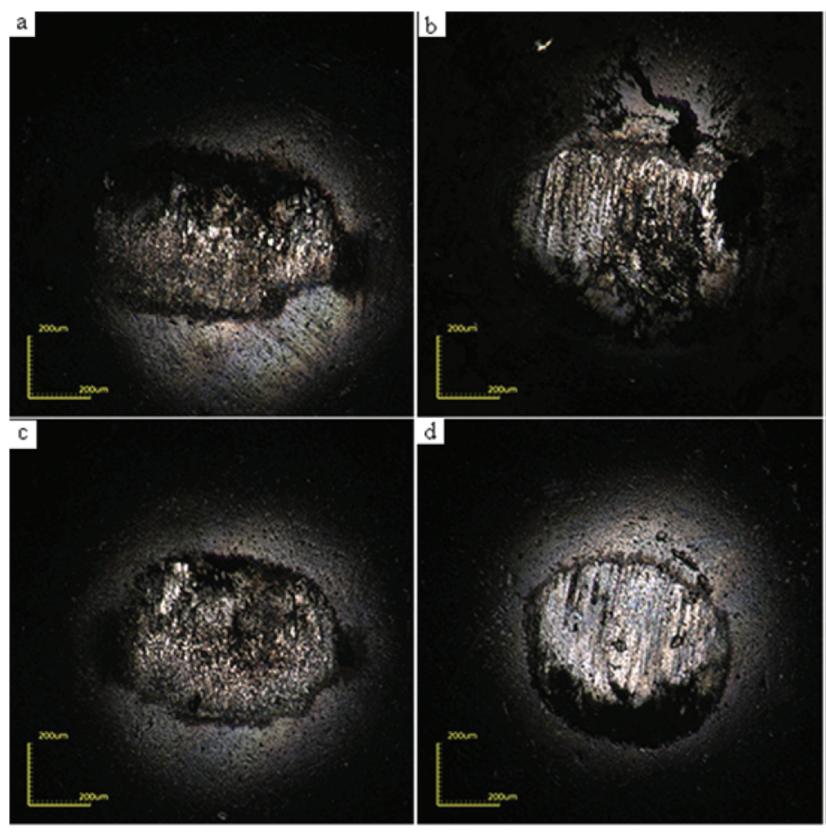

Figure 8: Wear morphology of different layer thickness: (a) $2 \mathrm{~mm}$, (b) $1.5 \mathrm{~mm}$, (c) $1 \mathrm{~mm}$, (d) $0.5 \mathrm{~mm}$.

the figure, as the same as wear morphology of sample's surface, the wear scar size for layer thickness over $1 \mathrm{~mm}$ is bigger than that below $1 \mathrm{~mm}$. It is analyzed that the Snbased solid solution was soft and even melted because of the large amount of friction heat, increasing the contact area between the grinding ball and sample's surface. While the layer thickness is below $1 \mathrm{~mm}$, especially $0.5 \mathrm{~mm}$, the surface microhardness is so large that it is very difficult to take place plastic deformation. Therefore, the wear rate of the thinner layer tin-based babbit is far below thicker layer tin-based babbit, and the service life of the thinner layer is longer than that too. What's more, according to the previous research, due to the large amount of friction heat, the grinding ball occurred oxidized reaction, the local wear scar was black and grown.

\section{Microstructure and formation mechanism of IMCs}

It is known to us all that the wear property and microhardness of tin-based babbit are dependent on its microstructure characteristics. As seen in Figure 9, the SbSn cubics are clearly identified in the $\mathrm{Sn}$ matrix, and $\mathrm{Cu}_{6} \mathrm{Sn}_{5}$ intermetallic compounds which distribute in the $\mathrm{Sn}$ matrix, have characteristic star shapes that can also be easily identified. However, with the layer thickness decreasing to $0.5 \mathrm{~mm}$, the microstructure of tin-based babbit mainly consists of large amount of SbSn and their grain boundary connected, which would apparently

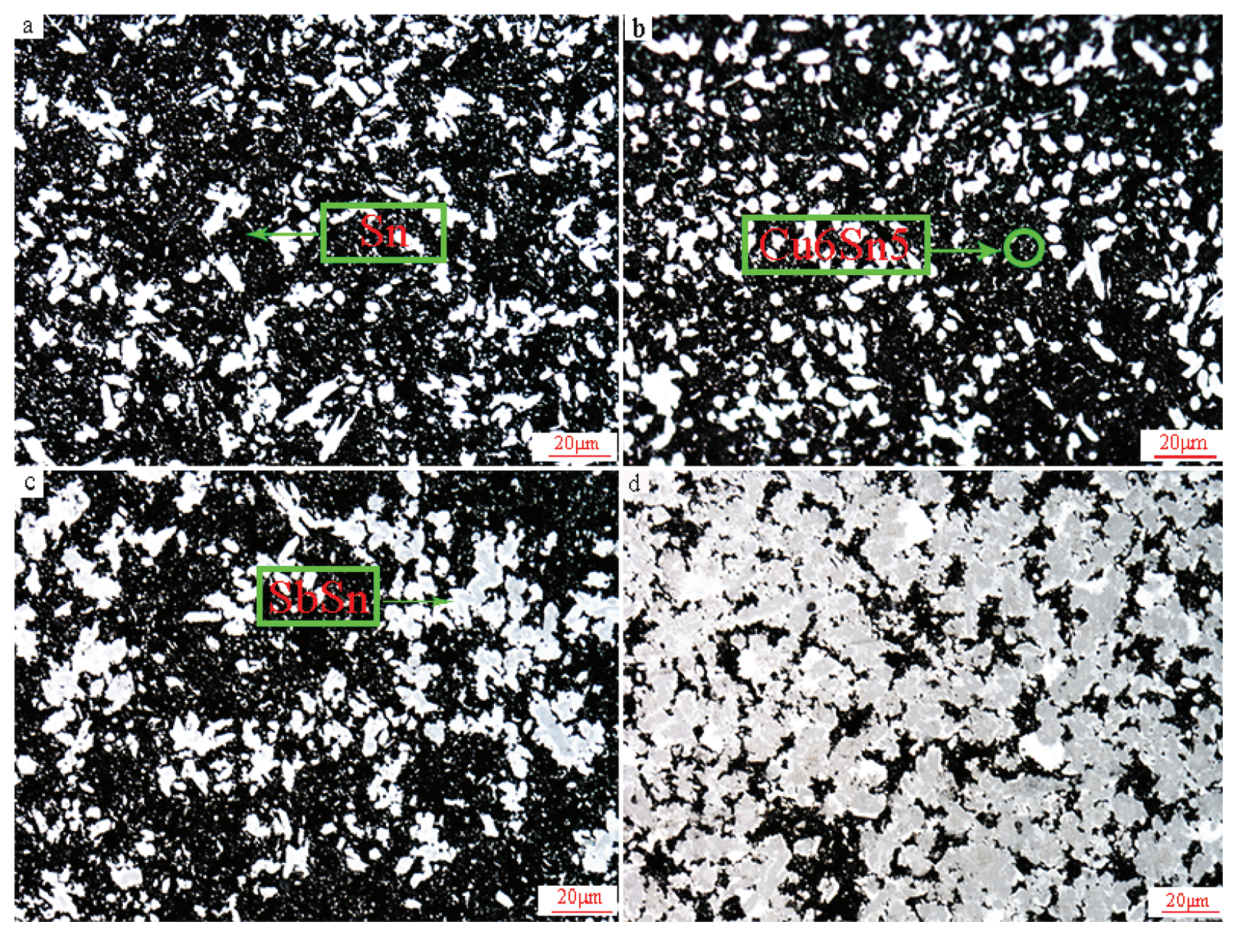

Figure 9: Microstructure of tinbased babbit at different layer thickness: a) $2 \mathrm{~mm}$, b) $1.5 \mathrm{~mm}$, c) $1 \mathrm{~mm}$, d) $0.5 \mathrm{~mm}$. 
improve the microhardness and antifriction property of layer's surface. It is analyzed that since the solidification temperature of $\mathrm{Cu}_{6} \mathrm{Sn}_{5}$, SbSn and Sn-based solid solution is $375^{\circ} \mathrm{C}, 273^{\circ} \mathrm{C}[14]$ and $230^{\circ} \mathrm{C}, \mathrm{Cu}_{6} \mathrm{Sn}_{5}$ compounds are firstly formed by over eutectic reaction [15] and precipitate from the melting tin-based babbit and distribute like star shape, then SbSn compounds are formed through monotectic reaction and precipitate from the melting tin-based babbit and distribute like cubic, finally Snbased solid solution precipitate from the melting metal during solidification process. The size of SbSn in Figure 9 (d) is larger than that in Figure 9 (a-c), increasing the microhardness of tin-based babbit. On the other hand, when the layer thickness was $0.5 \mathrm{~mm}$, Fe from substrate would migrate to tin-based babbit, which also affect the wear property and microhardness.

To further understand the reason that the wear property increased at thinner layer thickness and whether the Fe elements diffused to interfacial layer, the XRD analysis was carried out to identify the phase types and results were shown in Figure 10. It can be seen that tin-based babbit at $0.5 \mathrm{~mm}$ thickness not only was composed of Snbased solid solution, $\mathrm{SnSb}$ and $\mathrm{Cu}_{6} \mathrm{Sn}_{5}$, but also consisted of $\mathrm{Fe}, \mathrm{Fe}_{3} \mathrm{Sn}, \mathrm{FeSn}_{2}$, FeSn [8, 14].

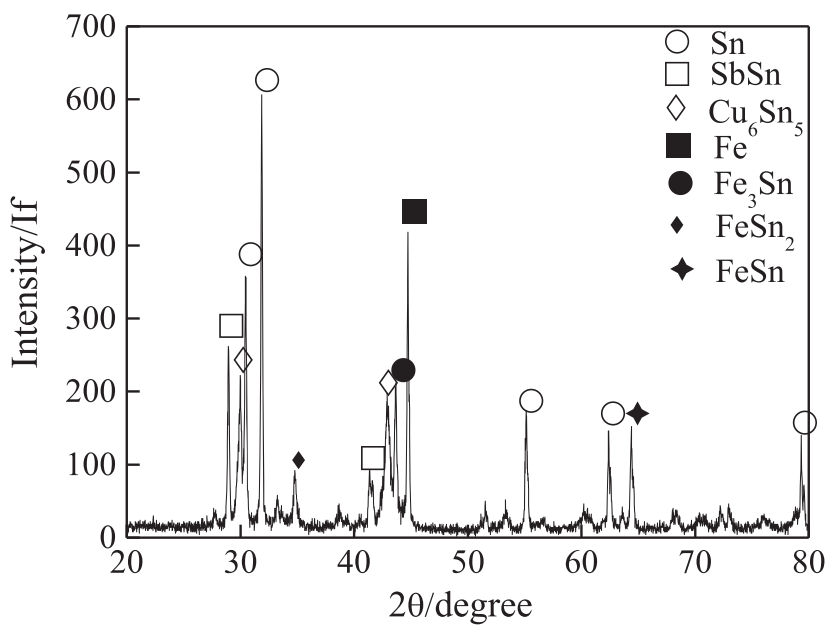

Figure 10: XRD analysis of IMCS.

In order to understand the IMCs formation mechanism, the SEM and EDS were carried out and the results were shown in Figure 11. Besides, the schematic illustrations of IMCs are established to analyze the formation process shown in Figure 12 (a-d). It can be seen from the figures, the surface of the substrate was
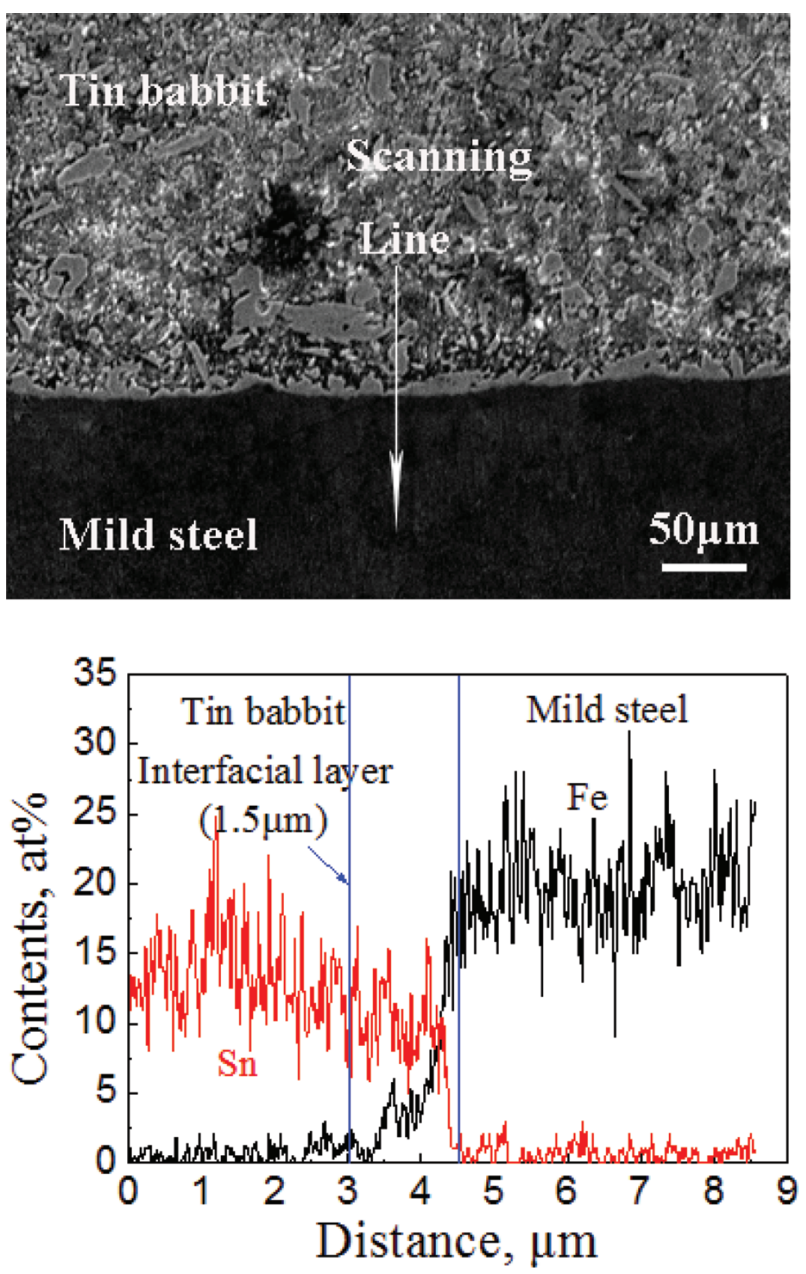

Figure 11: SEM and EDS analysis of IMCS.

heated by TIG arc and the surface temperature rise, which would lead to solid iron atomization of the substrate surface at the beginning stage, as shown in Figure $12(a-b)$. At the same time, interdiffusion of iron atoms and $\mathrm{Sn}$ atoms occurs when the Sn atoms and iron atoms contact physically. But diffusion of coefficient of iron atoms in the liquid tin-based babbit is much larger than that of tin-based babbit Sn atoms in solid mild steel [16], what's more the space of liquid atomic is larger than that of solid atomic. Therefore, iron atoms would diffuse to a relatively long distance into the liquid tin-based babbit layer, while most of Sn atoms are accumulated in the narrow band in the steel side at the interface and resulted in the formation of IMCs phased. Since the diffusion coefficient of iron atoms in Fe-Sn IMCs layer is very small, the IMCs seriously hinder the further diffusion of iron atoms 

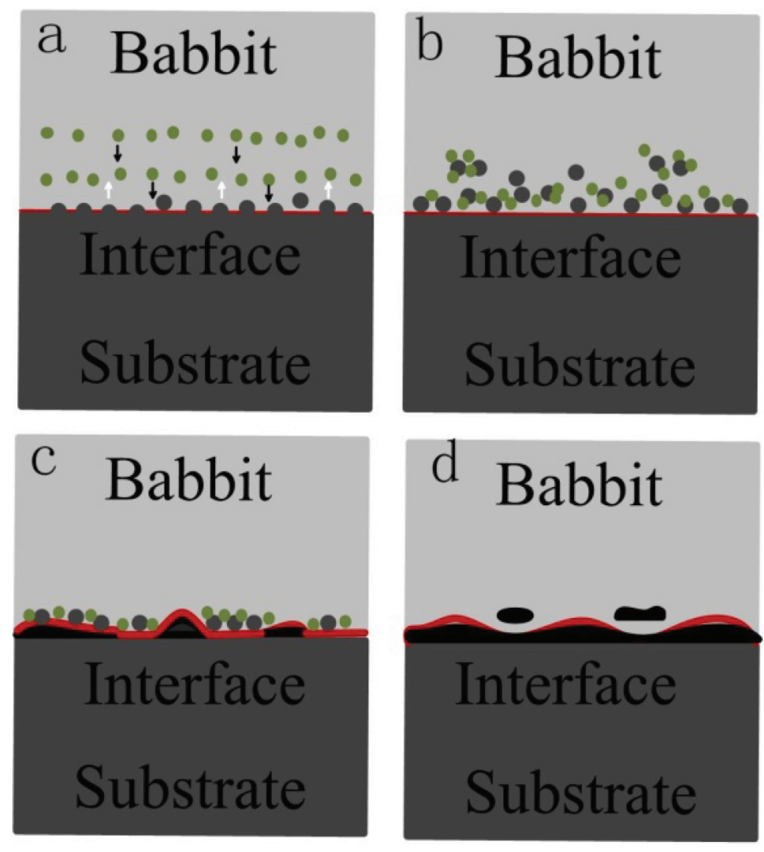

- $\mathrm{Fe}$

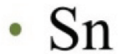

Figure 12: Formation mechanism of IMCs.

from the mild steel side into the liquid Tin-based babbit side. The solubility in liquid tin-based babbit deceased, iron atoms in the liquid tin-based babbit precipitate out in the form of intermetallic compounds and form a dense and unequal thickness Fe-Sn IMCs layer at the interface during the solidification of the liquid tin-based babbit, as shown in Figure 12 (c-d). Moreover, some IMCs in the interface is separated out due to the dynamic arc generating temperature gradient and the liquid deposited metal is stirred, leading to the free IMCs dissolute into the liquid tinbased babbit.

\section{Conclusions}

1. The tin-based babbit was prepared by the method of TIG arc brazing. In the brazed tin-based babbit, SbSn monotectic phase and $\mathrm{Cu}_{6} \mathrm{Sn}_{5}$ eutectic were formed.
When the layer thickness decreasing was $0.5 \mathrm{~mm}$, the wear rate the minimum and the microhardness was the largest.

2. Elements distribution analysis showed that Fe from mild steel transited and diffused into the brazing tinbased babbit. Meanwhile metallurgical reaction was occurred between Fe element and Sn element from tinbased babbit.

3. X-ray diffraction analysis illustrated that the brazing tin-based babbit was not only consisted of phase $\alpha$, phase $\beta$ (SnSb compound) and phase $\varepsilon$, but also was composed of $\mathrm{Fe}, \mathrm{Fe}_{3} \mathrm{Sn}, \mathrm{FeSn}$ and $\mathrm{FeSn}_{2}$.

Funding: The project was supported by the Science Research Project of Jiangsu Province, China (No.15KJA460006).

\section{References}

[1] L.I. Simma, Chem. Pet. Eng., 45 (2009) 649-652.

[2] Y.S. Korobov, S.V. Nevezhin and M.A. Filippov, J. Frict. Wear., 33 (2012) 190-194.

[3] A. Zeren, E. Feyzullahoglu and M. Zeren, Mater. Des., 28 (2007) 318-323.

[4] E. Feyzullahoglu, A. Zeren and M. Zeren, Mater. Des., 29 (2008) 714-720.

[5] S.B. Lin, J.L. Song, C.L. Yang, et al, Mater. Des., 509 (2009) 3140.

[6] C. Xu, G.M. Sheng, H. Wang, et al, J. Mater. Sci. Tech., 32 (2016) 167-171.

[7] S.B. Lin, J.L. Song, C.L. Yang, et al, Mater. Des., 31 (2010) 2637-2642.

[8] H. Giefers and M. Nicol, J. Alloy. Compd., 422 (2006) 132-144.

[9] W.D. Zhu, Mod. Mach., 1 (1994) 25-29.

[10] J. Tan, J.M. Chen and M. Liu, J. Mech. Eng., 47 (2011) 95-103.

[11] D.Y. He, B.Y. Fu and J.M. Jiang, Tribol, 27 (2007) 116-120.

[12] N.C. Kaushik and R.N. Rao, Tribol Int., 102 (2016) 52-60.

[13] C.Y. Wang, X.D. Li, Y. Chang, et al, Wear, 362 (2016) 121-128.

[14] A.K. Valeeva, I.S. Valeev and R.F. Fazlyakhmetov, J. Frict. Wear, 35 (2014) 311-315.

[15] R.Z. Tang and R.Z. Tian, Binary Alloy Phase Diagrams and Crystal Structure of Intermediate Phase, Central South University Press, (2009), p. 454.

[16] R.A. Ellis, S.D. Forder, T.H. English, et al, Hyperfine. Interact., 141 (2002) 447-451. 